\title{
Methylthioalkylmalate synthases: genetics, ecology and evolution
}

\author{
Markus Benderoth • Marina Pfalz • \\ Juergen Kroymann
}

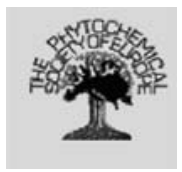

Received: 30 January 2008/Accepted: 10 April 2008/Published online: 31 May 2008

(C) The Author(s) 2008

\begin{abstract}
Glucosinolates display an enormous amount of structural variation, both within and between species. This diversity is thought to have evolved in response to challenges imposed on plants by their biotic environment. During the past decade, glucosinolates and myrosinase-catalyzed glucosinolate hydrolysis have become excellent examples for understanding functional diversification in plant secondary metabolism and plant defence. Methylthioalkylmalate (MAM) synthase genes and enzymes are central to the diversification of aliphatic glucosinolate structures in Arabidopsis thaliana and related plants. This review summarizes efforts to elucidate how $M A M$-mediated diversity in aliphatic glucosinolate structures is generated and maintained. It also attempts to put variability in methionine carbon chain elongation during glucosinolate biosynthesis into an ecological and evolutionary context.
\end{abstract}

Keywords Complex traits - Evolutionary dynamics - Glucosinolate metabolism .

Natural variation $\cdot$ Plant-insect interactions

The authors M. Benderoth and M. Pfalz have contributed equally to the manuscript.

M. Benderoth $\cdot$ M. Pfalz $\cdot$ J. Kroymann $(\square)$

AG Genetics and Evolution, Max Planck Institute for Chemical Ecology, Hans-Knöll-Str. 8, 07745 Jena, Germany

e-mail: kroymann@ice.mpg.de
Abbreviations
IPMS Isopropylmalate synthase
MAM Methylthioalkylmalate synthase
NIL Near-isogenic line
QTL Quantitative trait locus/loci
RIL Recombinant inbred line

\section{Introduction}

Glucosinolates and myrosinases are almost exclusively found in plants from the order Capparales. This order consists of more than a dozen families, including the Brassicaceae and the Capparaceae (Rodman 1991a, b; Rodman et al. 1996). More than 130 different glucosinolates have been characterized; however glucosinolate composition varies remarkably between and within species (Daxenbichler et al. 1991; Fahey et al. 2001; Kliebenstein et al. 2001a; Windsor et al. 2005; Heidel et al. 2006). In Arabidopsis thaliana, nearly 40 different glucosinolates have been identified (Kliebenstein et al. 2001a; Reichelt et al. 2002). These glucosinolates are generated from methionine, tryptophan or phenylalanine, with methionine-derived (aliphatic) glucosinolates being the predominant glucosinolate class represented in A. thaliana.

Comparative analyses of glucosinolate profiles among Arabidopsis accessions reveal extensive qualitative and quantitative variation (Kliebenstein et al. 2001a; Pfalz et al. 2007). Quantitative trait locus 
(QTL) mapping studies in various Arabidopsis recombinant inbred line (RIL) populations (Magrath et al. 1994; Mithen et al. 1995; Campos de Quiros et al. 2000; Kliebenstein et al. 2001b, c; Keurentjes et al. 2006; Wentzell et al. 2007) show consistently that two major genetic loci, methylthioalkylmalate (MAM) and $A O P$ (Fig. 1), account for most of the variability in aliphatic glucosinolate structures. Enzymes encoded at $A O P$ are responsible for the modification of the side chain structure (Kliebenstein et al. 2001b), while the MAM locus (also referred to as ELONG, GS-ELONG or GSL-ELONG) controls variability in aliphatic glucosinolate carbon chain length.

MAM encodes a small family of MAM synthases (Kroymann et al. 2001, 2003; Benderoth et al. 2006) in A. thaliana and related Brassicaceae. MAM synthases catalyze the condensation of $\omega$-methylthio-2-oxoalkanoic acids derived from methionine with acetyl-CoA to form methylthioalkylmalic acids. Subsequent isomerization and oxidative decarboxylation reactions lead to the generation of $\omega$-methylthio-2-oxoalkanoic acids with extended carbon chains (Fig. 2). Together, condensation, isomerization and oxidative decarboxylation are referred to as the methionine carbon chain elongation cycle, with an extension of one methylene group per cycle. MAM substrate specificity (Table 1) determines whether the reaction products of a given cycle enter the biosynthetic pathway generating the glucosinolate core structure or whether they undergo additional cycles of carbon chain elongation. A. thaliana MAM1 and MAM2 are involved in the formation of aliphatic glucosinolates with short carbon chains (Kroymann et al. 2001; Textor et al. 2004; Benderoth et al. 2006), while MAM3 catalyzes condensation reactions in the biosynthesis of aliphatic glucosinolates with long carbon chains (Field et al. 2004; Textor et al. 2007; Knoke et al. 2008). MAM synthases determine variability during the earliest stage of aliphatic glucosinolate biosynthesis and play a central role in glucosinolate diversity. Hence, evolutionary forces acting on MAM have a fundamental impact on aliphatic glucosinolate composition.

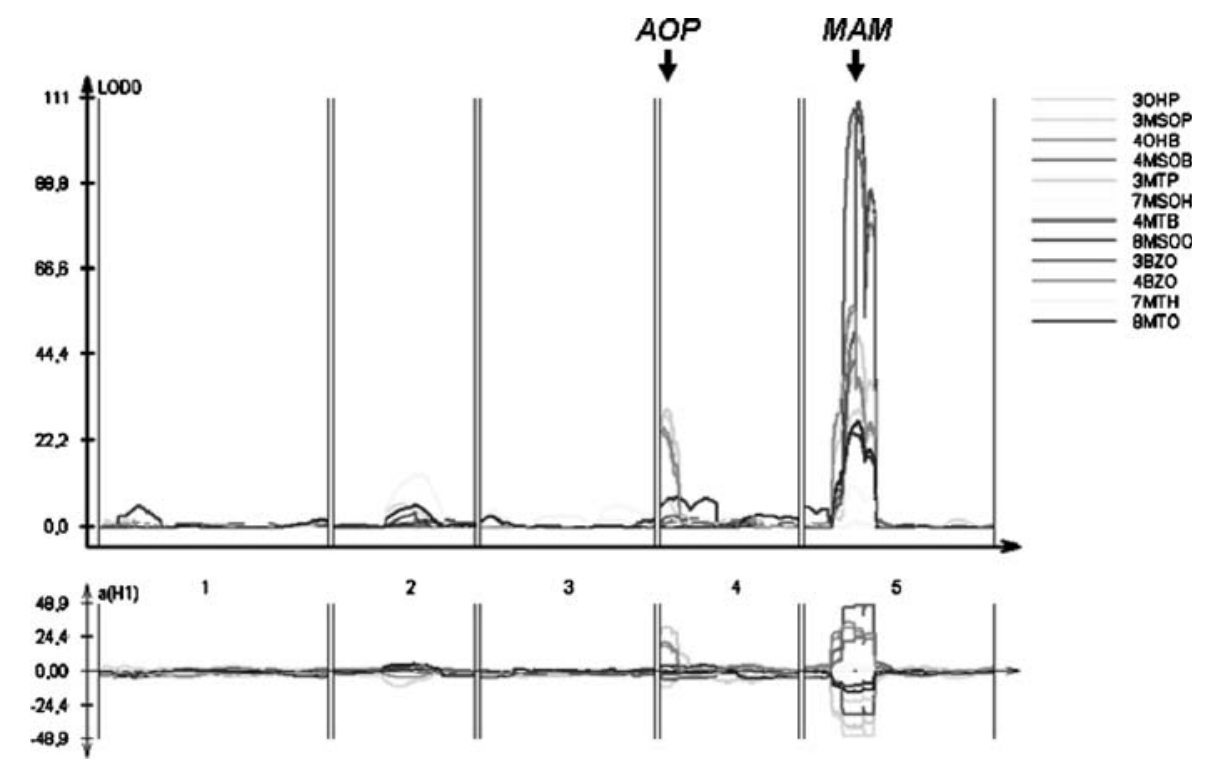

Fig. 1 QTL for aliphatic glucosinolates in seeds from Col-0 $\times$ Ler RILs. Top: The MAM locus on chromosome five influences the quantity of all aliphatic glucosinolates. 3-hydroxypropyl (3OHP), 3-methylsulfinylpropyl (3MSOP), 3-methylthiopropyl (3MTP) and 3-benzoyloxypropyl (3BZO) originate from homomethionine (3C), 4-hydroxybutyl (4OHB), 4-methylsulfinylbutyl (4MSOB), 4-methylthiobutyl (4MTB) and 4-benzoyloxybutyl (4BZO) from dihomomethionine (4C), 7-methylsulfinylheptyl (7MSOH) and 7-methylthioheptyl (7MTH) from pentahomomethionine (7C) and 8-methylsulfinylocytyl
(8MSOO) and 8-methylthiooctyl (8MTO) from hexahomomethionine (8C). Bottom: Additive genetic effects for homo- and pentahomomethionine-derived glucosinolates are opposite to those for dihomo- and hexahomomethionine-derived glucosinolates. QTL mapping was conducted with Windows QTL cartographer 2.5 (Wang et al. 2001-2004) and is based on log-transformed HPLC data from 297 Col-0 × Ler RILs (John D'Auria and Juergen Kroymann, unpublished data). For all traits, the significance threshold was $\sim 2.5$ LOD units, based on 1,000 permutations of the data 


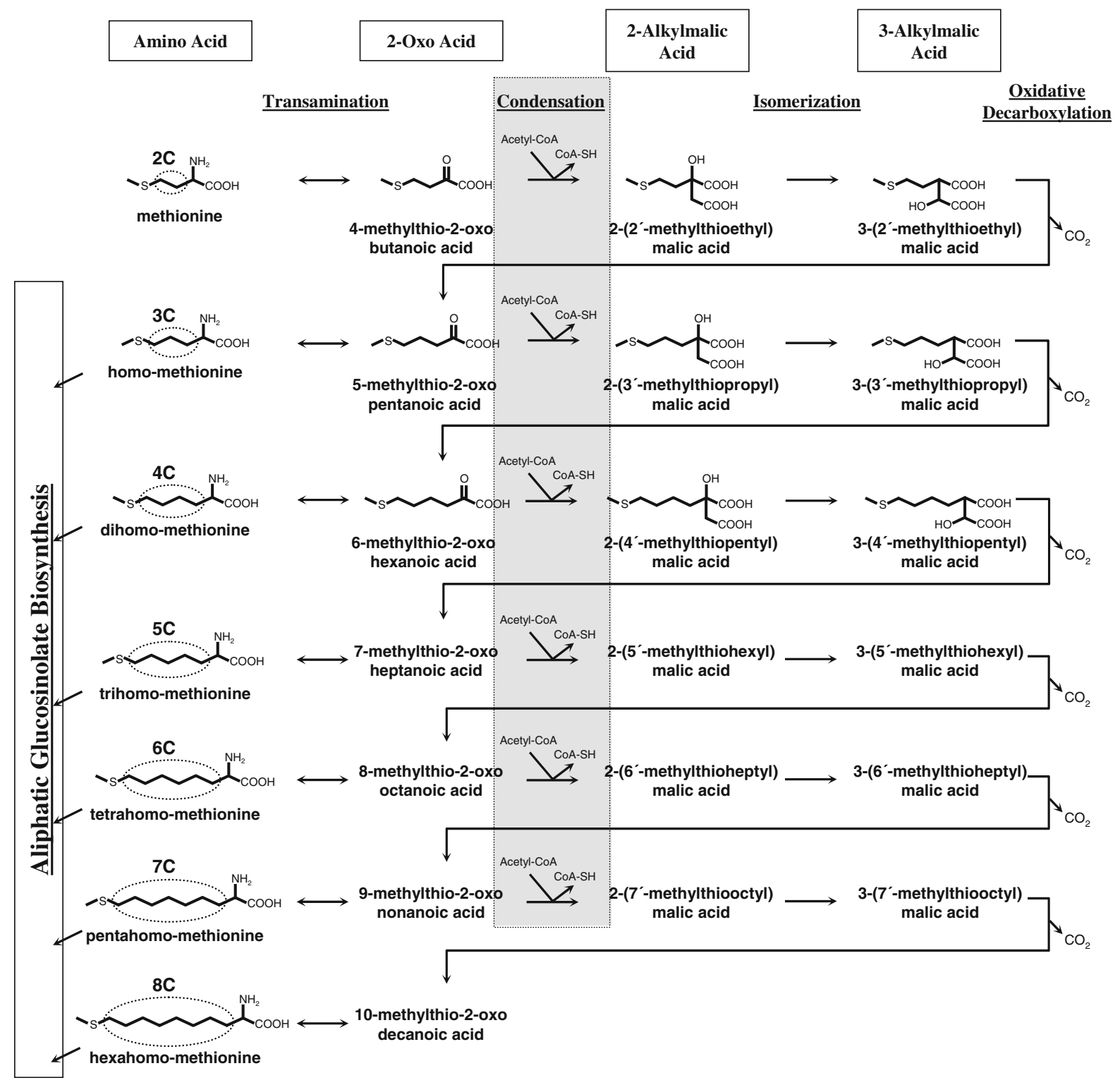

Fig. 2 The methionine carbon chain elongation cycle. MAM synthases encoded at the MAM locus catalyze the committed step in methionine carbon chain elongation. Two subsequent reactions, an isomerization and an oxidative decarboxylation complete a cycle. MAM substrate specificity (Table 1)

\section{Cloning of the MAM QTL}

The widely used A. thaliana accession Landsberg erecta (Ler) accumulates homomethionine-derived (3C) glucosinolates. In contrast, aliphatic glucosinolates in Columbia (Col-0), recognized for having provided the blueprint of the Arabidopsis genome determines whether $\omega$-methylthio-2-oxoalkanoic acids remain in the carbon chain elongation cycle or enter the biosynthetic pathway that generates the glucosinolate core structure. Here, all reactions occurring in A. thaliana are depicted; in other Brassicaceae fewer or more cycles are possible

(Arabidopsis Genome Initiative 2000), originate mainly from dihomomethionine (4C). This biochemical difference between Col-0 and Ler enabled initial mapping of the responsible genetic locus to a region of approximately $140 \mathrm{~kb}$ on chromosome 5 (Magrath et al. 1994; Campos de Quiros et al. 2000). Within this region, two tandemly arranged genes were identified as 
Table 1 MAM substrate specificities in glucosinolate biosynthesis
Assays were conducted with heterologously expressed $M A M$ genes and generic and/or artificial substrates

\begin{tabular}{|c|c|c|c|}
\hline MAM synthase & Substrates & Elongation cycle(s) & References \\
\hline A. thaliana MAM1 & $2 \mathrm{C}, 3 \mathrm{C},(4 \mathrm{C})$ & $1,2,(3)$ & $\begin{array}{l}\text { Kroymann et al. (2001), } \\
\text { Textor et al. (2004), } \\
\text { Benderoth et al. (2006) }\end{array}$ \\
\hline A. thaliana MAM2 & $2 \mathrm{C}$ & 1 & Benderoth et al. (2006) \\
\hline A. thaliana MAM3 & $2 \mathrm{C}-7 \mathrm{C}$ & $1-6$ & $\begin{array}{l}\text { Textor et al. (2007), } \\
\text { Knoke et al. (2008) }\end{array}$ \\
\hline A. lyrata MAMa & $2 \mathrm{C}$ & 1 & Benderoth et al. (2006) \\
\hline B. stricta MAMa & & & \\
\hline
\end{tabular}

candidates, based on their sequence similarity with isopropylmalate synthase (IPMS) genes. IPMS catalyzes the condensation of 2-oxoisovalerate with acetylCoA to form isopropylmalate in leucine biosynthesis, a reaction similar to the MAM-catalyzed condensation of $\omega$-methylthio-2-oxoalkanoic acids with acetyl-CoA in glucosinolate biosynthesis. The two candidate genes were termed $M A M 1$ and $M A M-L$ (meanwhile often referred to as $M A M 3$ ), respectively (Kroymann et al. 2001).

In the Col-0 accession, MAM1 and MAM3 are separated by ca. $11.5 \mathrm{~kb}$ of intervening sequence. High-resolution mapping was employed to separate functional effects of these candidate genes. Col- 0 was crossed with CL5, an RIL from the Col-0 $\times$ Ler population (Lister and Dean 1993). This particular line was chosen because it shared approximately $70 \%$ of its genome with the Col- 0 accession but has the Ler allele at the MAM locus, thus enabling fine-mapping with near-isogenic lines (NILs). In Col-0 $\times$ CL5 F 2 progeny one line was identified that had recombined between MAMI and MAM3. This recombinant line had the Col-0 MAM1 genotype and was heterozygous at MAM3. Its glucosinolate phenotype resembled the parental Col-0 profile closely, with $4 \mathrm{C}$ glucosinolates predominating. Likewise, progeny from this line produced mainly $4 \mathrm{C}$ glucosinolates when they had the Col-0 MAM1 and the Ler MAM3 genotype. Hence, the biochemical difference in short-chain aliphatic glucosinolate composition was attributable to MAM1 and not MAM3 (Kroymann et al. 2001). Further evidence for the role of MAM1 in methionine carbon chain elongation was obtained with MAM1 mutants, deficient in dihomomethionine-derived glucosinolates (Haughn et al. 1991), and with biochemical assays of heterologously expressed $M A M 1$, which showed that the encoded protein has the capacity of condensing $\omega$-methylthio-2-oxoalkanoic acids with acetyl-CoA
(Kroymann et al. 2001; Textor et al. 2004; Benderoth et al. 2006).

\section{Origin of $M A M$ genes}

Several groups of enzymes catalyze condensation reactions between 2-oxo acids and acetyl-CoA. These enzymes belong to enzyme class EC 2.3.3.-. Examples are citrate synthase (EC 2.3.3.1), which condenses oxaloacetate with acetyl-CoA in the TCA cycle, malate synthase (EC 2.3.3.9), which is responsible for the condensation of glyoxylate with acetyl$\mathrm{CoA}$ in the glyoxylate cycle, and 2-IPMS (EC 2.3.3.13) involved in leucine biosynthesis.

Among the genes that encode enzymes of class EC 2.3.3.-, MAM genes share the highest degree of sequence similarity with 2-IPMS genes. Phylogenetic analyses reveal a close relationship between $M A M$ and IPMS genes, with MAMs having evolved from IPMS (Fig. 3). Similar gene structures of MAM and IPMS corroborate this close relationship; most intron positions are identical in MAM and IPMS (Kroymann et al. 2001). However, the first IPMS exon corresponds to the first two exons in MAM genes, and the last four IPMS exons are fused to a single exon in MAMs. Exon fusion was accompanied with a net loss of ca. $250 \mathrm{bp}$ in the $3^{\prime}$ portion of MAM genes, and may have functional significance since this region encodes a conserved allosteric leucine binding site in IPMS (de Kraker et al. 2007). As a consequence, Arabidopsis IPMS genes consist of 12 exons and $M A M$ genes of 10 exons, with IPMS exons 2-8 and MAM exons 3-9 corresponding to one another (Fig. 4). Furthermore, all MAM genes encode an N-terminal plastid targeting signal (Kroymann et al. 2001), suggesting that methionine carbon chain elongation occurs-like leucine biosynthesis (Hagelstein and Schultz 1993)—in the plastids, a 


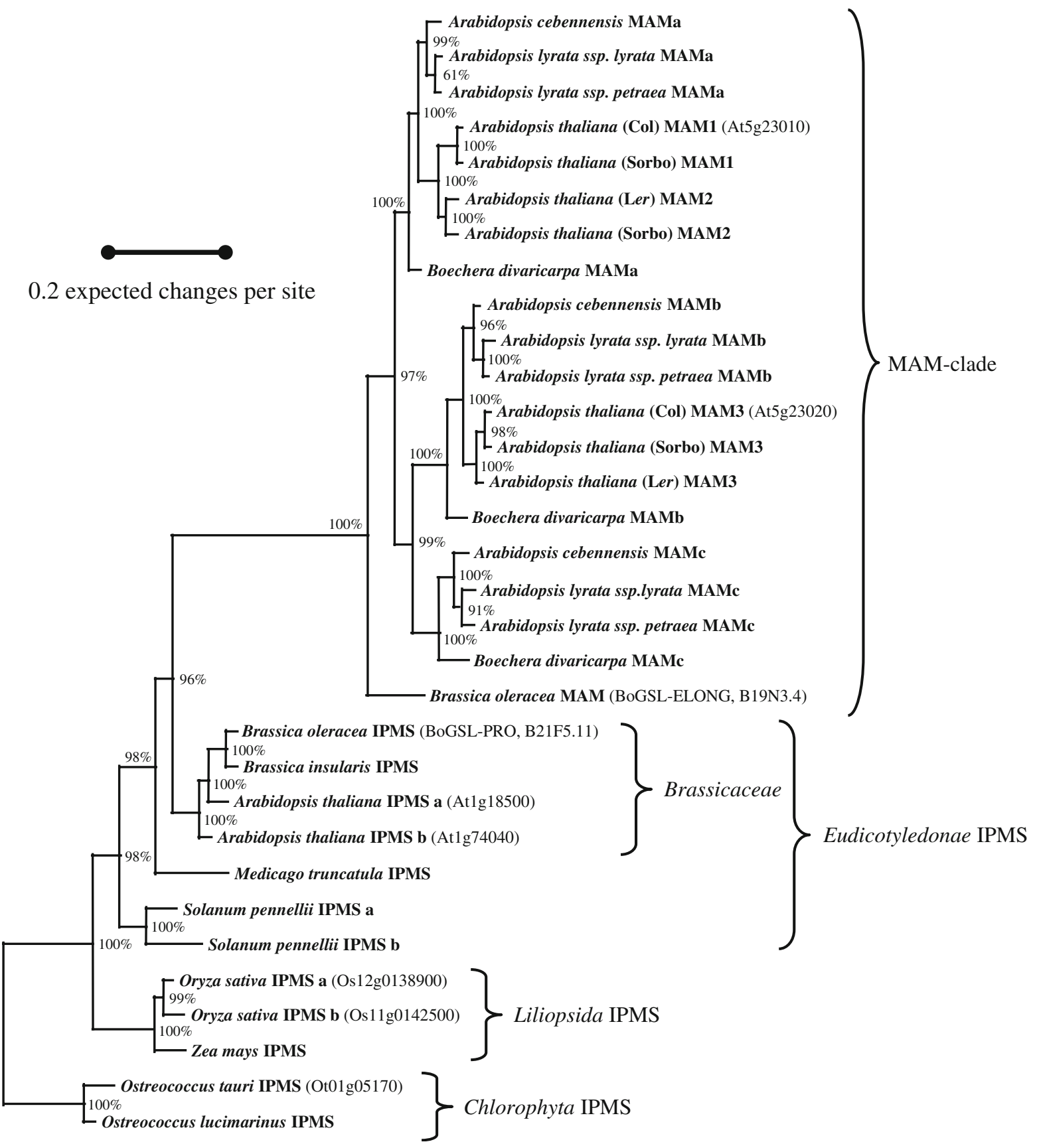

Fig. 3 Bayesian phylogenetic analysis of MAM and IPMS sequences. Amino acid sequences were aligned with ClustalW. Target peptides and alignment gaps were excluded. The tree was constructed with MrBayes v 3.1.2 (Huelsenbeck and Ronquist 2001) using a mixed amino acid substitution model approach. Data structure was best explained by the wag-model (Whelan and Goldman 2001) with a posterior probability of 1.000. The Markov-Chain-Monte-Carlo simulation was performed in three parallel runs with four chains each for $1,000,000$ iterations with a sample frequency of 200 and a burnin fraction of 0.05 . Convergence diagnostic over all three runs (PSRF) was 1.000. Shown are posterior probabilities for all internal nodes. Note that Medicago truncatula IPMS is incorrectly annotated in the databases; here, a corrected annotation was assembled from clone mth2-19b5 for alignment and tree construction 
Fig. 4 Exon-intron structure of IPMS and MAM genes. IPMS genes from higher plants consist of 12 , $M A M$ genes of ten exons. Differences between Arabidopsis IPMS and $M A M$ gene structures involve the split of IPMS exon I (*), the fusion and partial loss of IPMS exons $\mathrm{IX}, \mathrm{X}$ and $\mathrm{XI}(* *)$, and the loss of IPMS exon XII $(* * *)$. Plastid targeting sequences are represented by dashed lines. Os, Oryza sativa; Bo, B. oleracea; Col, A. thaliana accession Col-0

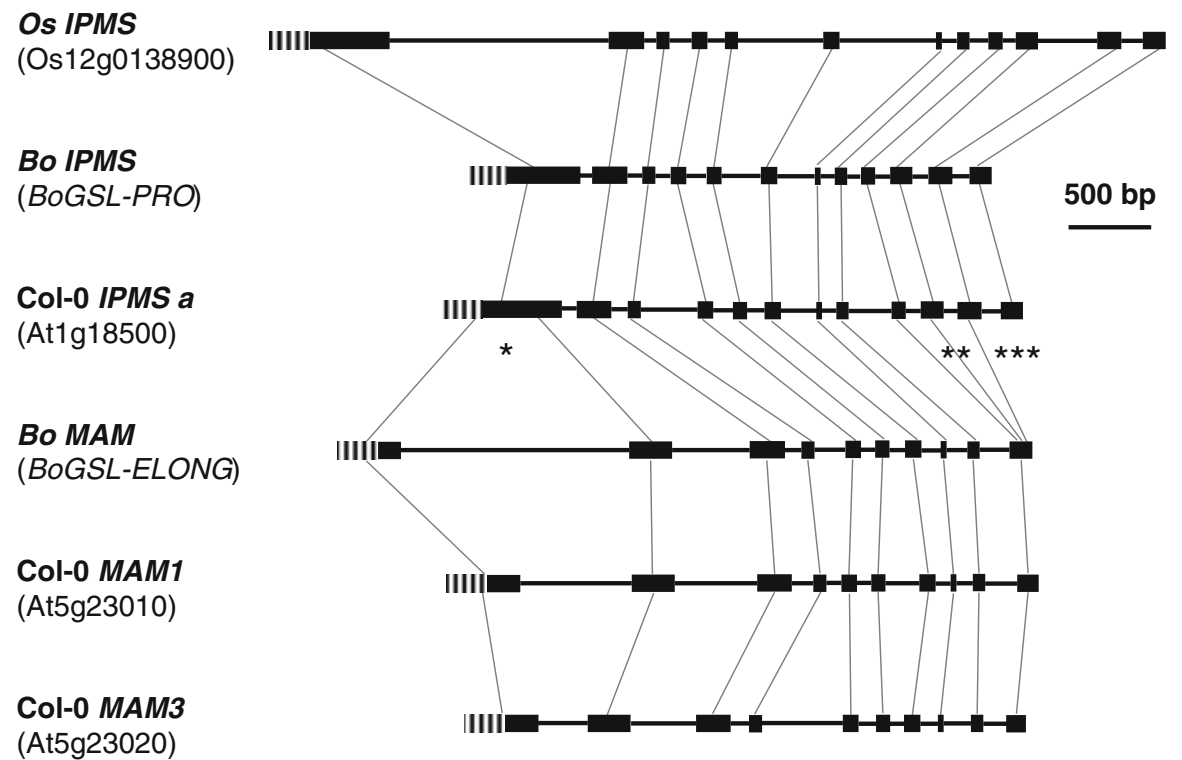

comparison enabled the reconstruction of the archetypical configuration of the MAM locus in A. thaliana, consisting of tandemly arranged MAM2, MAM1 and $M A M 3$ genes, all transcribed in the same direction. However, secondary gene deletions have occurred frequently in the history of the A. thaliana MAM cluster. In some accessions (including Col-0) the $M A M 2$ gene was deleted. In other accessions, the $M A M 1$ gene was deleted, either partially, involving promoter and $5^{\prime}$-portion of the coding sequence (e.g. in Ler), or completely. Finally, the Lm-2 accession contains a chimerical gene with $5^{\prime}$ MAM2 fused to $3^{\prime}$ $M A M 1$ sequence, possibly caused by a deletion of the intervening region. Nonetheless, in contemporary accessions at least one of these genes has been retained. Likewise, all tested natural accessions contain a functional MAM3 gene.

Sequence exchange between MAM1 and MAM2 genes is another factor contributing to variability in the gene composition of the MAM locus. In some accessions, sequence transfer has occurred from $M A M 2$ to $M A M 1$, in others sequence portions have been shifted from MAM1 to MAM2. In the most extreme cases, represented by two accessions from Tajikistan (Hodja and Condara), the gene at the position originally occupied by $M A M 1$ has been almost completely converted to a MAM2-like gene. However, another accession from Tajikistan, Sorbo, was not affected by gene deletion or conversion events. This accession has functional MAM2 and 


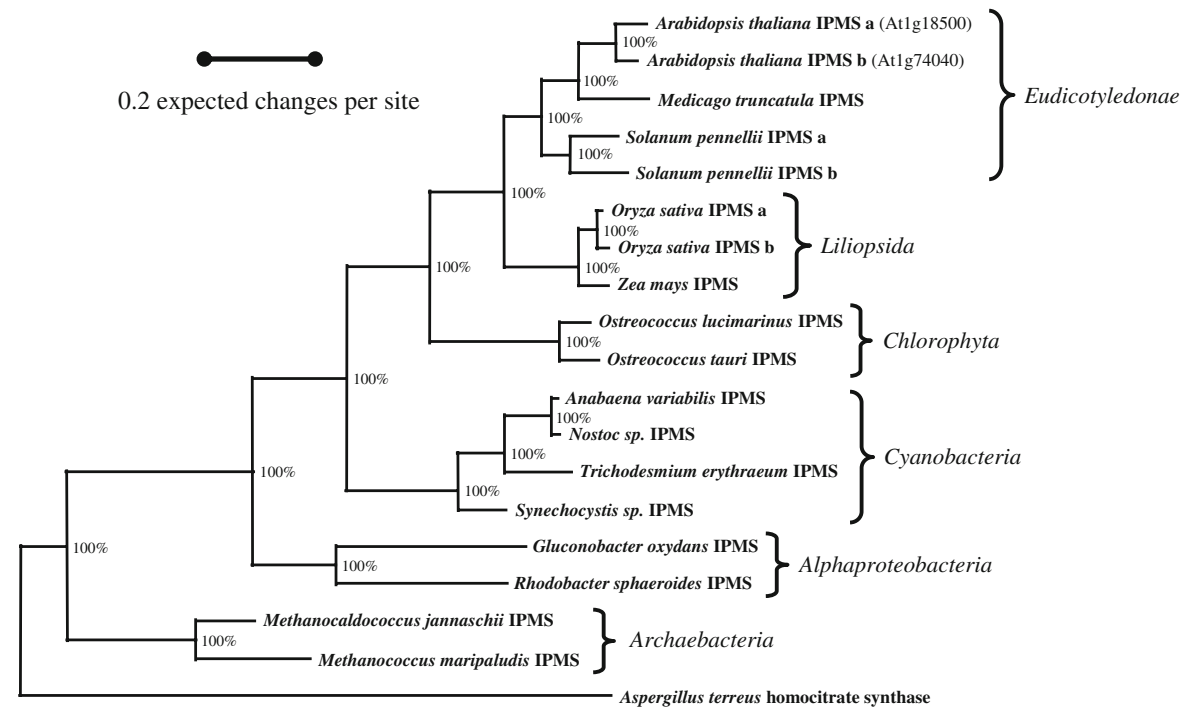

Fig. 5 Evolutionary origin of plant IPMS sequences inferred with Bayesian phylogenetic analysis. Calculations were based on ClustalW-aligned amino acid sequences. Target peptides and alignment gaps were excluded. The analysis was conducted as explained in the legend to Fig. 3. Again, data structure was best explained by the wag-model (Whelan and Goldman 2001) with a posterior probability of 0.999 . Convergence diagnostic over all three runs (PSRF) was 1.000. Posterior probabilities for all internal nodes are shown

larger quantities of homo- (3C) and hexahomomethionine (8C) derivatives. Moreover, the MAMI/MAM2 polymorphism also caused differences in the concentration of total aliphatic glucosinolates, with Ler MAM2 genotypes accumulating $60 \%$ more aliphatic glucosinolates in leaves and $20 \%$ more in seeds than Col-0 MAM1 genotypes (Kroymann et al. 2003; Fig. 8).

Even though MAM1 and MAM2 lack the capacity to catalyze the condensation reactions in advanced methionine carbon chain elongation cycles (Benderoth et al. 2006), the QTL peaked in all cases at an interval containing MAM1 or MAM2, but not MAM3. Hence, the MAM1/MAM2 polymorphism influenced not only aliphatic glucosinolates with short carbon chains but also affected glucosinolates with long carbon chains. The biochemical basis for this effect of MAM1 versus MAM2 on long-chain aliphatic glucosinolate accumulation is not yet understood.

A complex influence of the MAM locus on glucosinolate profiles was also seen in studies with another Arabidopsis RIL population, Bay- $0 \times$ Sha (Kliebenstein et al. 2006; Wentzell et al. 2007). eQTL mapping and network analysis (further explained in Kliebenstein 2008) suggest that this influence is, to a certain degree, caused by changes in transcript levels. 


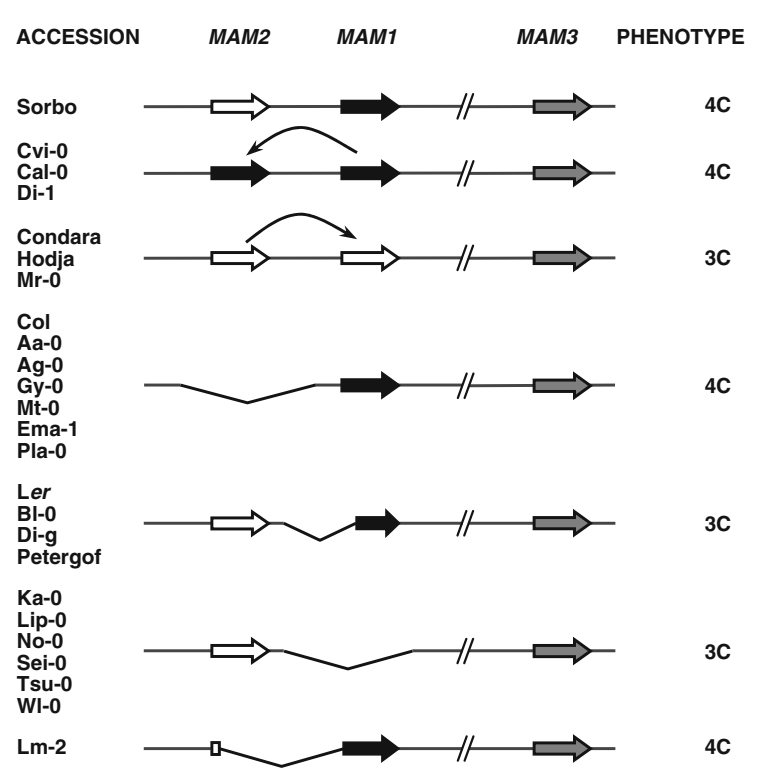

Fig. 6 Schematic representation of the $M A M$ cluster in Arabidopsis accessions. The organization of the $M A M$ cluster is highly variable. Partial or complete deletions of $M A M 2$ or MAM1 have occurred frequently. In Lm-2, 5'-MAM2 sequence is fused to $3^{\prime}$ $M A M 1$ sequence. Furthermore, sequence information has been transferred between loci. In Cvi-0, Cal-0 and Di-1, the gene at the $M A M 2$ position has been partially converted into a MAM1-like sequence; in Condara, Hodja and Mr-0, MAM1 has been converted into a MAM2-like gene. All tested accessions have a MAM3 gene. The predominant aliphatic glucosinolate class is indicated in the right column (3C: homomethionine-derived, $4 \mathrm{C}$ : dihomomethionine-derived)

\section{Ecological consequences of the MAM1/MAM2 polymorphism}

Two lepidopteran insects, Spodoptera exigua and Plutella xylostella, were tested for differences in larval performance contingent on the genotype at the $M A M$ locus in Col-0 $\times$ CL5 NILs. S. exigua is referred to as a generalist due to possession of a broad host range; it has the ability to feed on a variety of plants from different families. In contrast, P. xylostella has a narrow host range utilizing almost exclusively plants from the crucifer family. S. exigua was found to respond to the MAM1/MAM2 polymorphism. Its performance was lower on the MAM2 genotype, and larvae caused approximately $17 \%$ less damage than on the MAM1 genotype (Kroymann et al. 2003). Again, single marker analysis showed that the resistance QTL peaked in the interval containing MAMI/MAM2 but not MAM3 (Fig. 8).

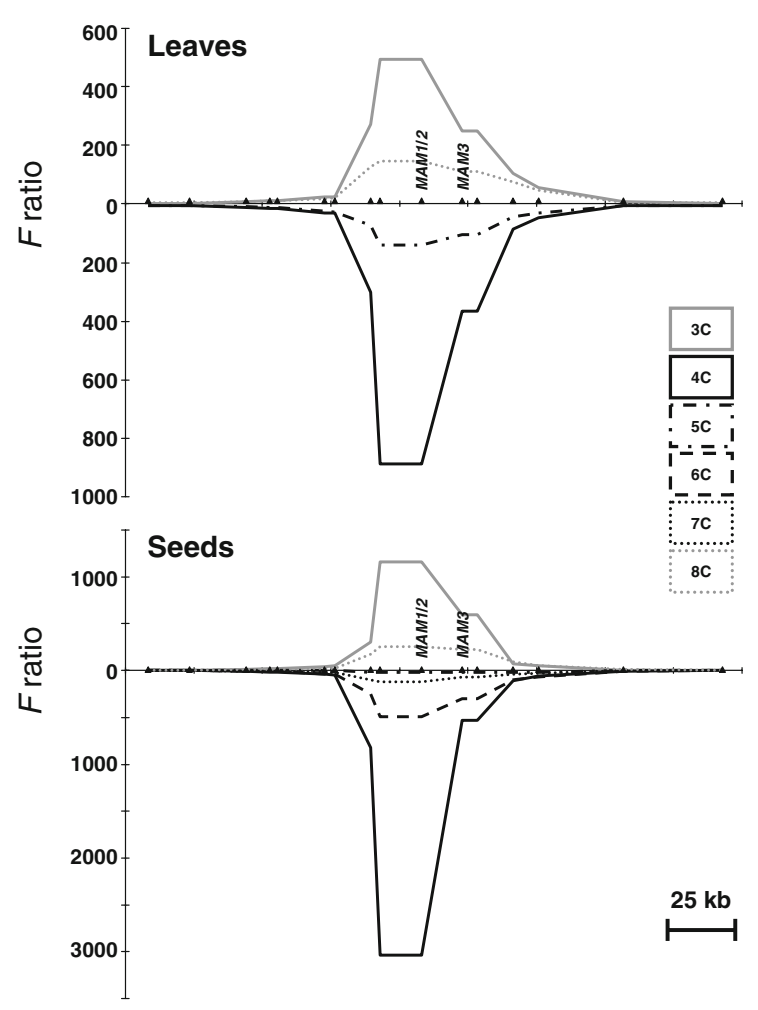

Fig. 7 Single-marker QTL analysis of aliphatic glucosinolates with different carbon chain lengths in a $205-\mathrm{kb}$ region of the Arabidopsis genome. Fifty-eight NILs with recombination breakpoints in the $M A M$ region were assayed for trait values in leaves (top) and seeds (bottom). QTL for homo- (3C), dihomo(4C), trihomo- $(5 \mathrm{C})$, tetrahomo- $(6 \mathrm{C})$, pentahomo- $(7 \mathrm{C})$ and hexahomomethionine (8C)-derived glucosinolates are centred at a $15-\mathrm{kb}$ non-recombinant region that contains Col-0 MAM1 or Ler MAM2, but not MAM3. Col-0 MAM1 genotypes produce more $4 \mathrm{C}-7 \mathrm{C}$ derivatives than Ler MAM2 genotypes. By contrast, Ler MAM2 genotypes accumulate higher quantities of $3 \mathrm{C}$ and $8 \mathrm{C}$ derivatives. Small black triangles show marker positions based on the Col-0 sequence; the position of MAM1/ $M A M 2$ and MAM3 is indicated. $F$ ratios quantify statistical significance of a QTL at a given marker position. Note that leaves contain lower concentrations of glucosinolates than seeds; therefore, low-abundant glucosinolates are difficult to detect by HPLC in leaves

For P. xylostella, no significant difference was found with ANOVA. Nonetheless, larvae performed on average better on MAM2 genotypes (Kroymann et al. 2003). Furthermore, re-analysis of this data set with regression showed that Plutella larval herbivory was positively correlated with leaf aliphatic glucosinolate content (Kliebenstein et al. 2005). Hence, the MAM1/ $M A M 2$ polymorphism had contrasting effects on S. exigua versus $P$. xylostella. MAM2 genotypes were 


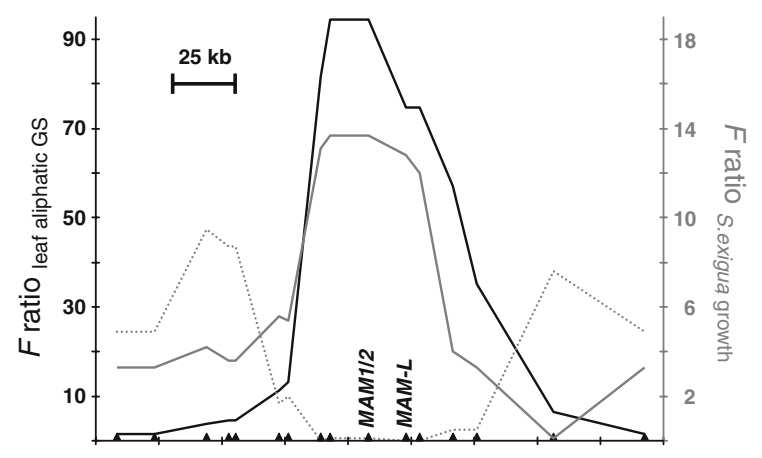

Fig. 8 Single-marker QTL analysis of leaf total aliphatic glucosinolates (black line), resistance against $S$. exigua (solid grey line) and growth rate (dotted grey line). QTL for leaf aliphatic glucosinolates and for resistance against $S$. exigua centre at a $15-\mathrm{kb}$ non-recombinant interval containing Ler $M A M 2$ or Col-0 MAM1. NILs with a Ler MAM2 accumulate more aliphatic glucosinolates and are more resistant against Spodoptera than the MAM1 genotype. Two QTL for biomass accumulation were detected upstream and downstream of the $M A M$ cluster, but there is no indication for a growth rate QTL at the MAM genes (Kroymann et al. 2003; Kroymann and Mitchell-Olds 2005)

better defended against the generalist insect herbivore, whereas MAM1 genotypes appeared to suffer less damage from the specialist. However, although the MAM1/MAM2 polymorphism controls these differences in insect performance, it is not yet clear which biochemical parameter ultimately accounts for the observed effects, due to the complex influence of the MAM locus on aliphatic glucosinolate profiles. Differences in resistance could be attributable to leaf total aliphatic glucosinolate concentration, levels of individual glucosinolates, differences in glucosinolate composition, or any combination of these factors.

Since MAM2 genotypes produced approximately $60 \%$ more leaf and $20 \%$ more seed glucosinolates than MAM1 genotypes, this raised the question whether increased glucosinolate production in the $M A M 2$ genotype was paid for with a reduction in plant growth rate. Such allocation costs can occur when defenses are energetically expensive, so that genotypes with strong defenses have fewer resources to invest in growth and reproduction (Purrington 2000; Tian et al. 2003). Therefore, dry weight of plant rosettes was measured at the pre-reproductive state. Quantitative analyses indicated the presence of growth rate QTL upstream and downstream of the $M A M$ locus, but there was no trace of a significant genotype effect on biomass accumulation at the MAM locus itself (Fig. 8, Kroymann et al. 2003; Kroymann and Mitchell-Olds 2005). Thus, allocation costs appear to be of minor importance for the MAM1/ MAM2 polymorphism in A. thaliana.

\section{Generation of glucosinolate diversity}

Sequencing of the MAM locus from the close A. thaliana relatives A. petraea ssp. lyrata (=A. lyrata), Arabidopsis petraea ssp. petraea (= A. petraea) and Arabidopsis cebennensis, and the more distant relative Boechera divaricarpa revealed that these species all possessed three MAM genes in the MAM cluster. These genes were termed MAMa, MAMb and MAMc (Fig. 9, Benderoth et al. 2006). Phylogenetic analyses showed that $A$. thaliana MAM1 and MAM2 originated from the duplication of a MAMa gene after $A$. thaliana diverged from its congeners (Fig. 3). $M A M b$ genes were found to be orthologous to A. thaliana MAM3. A MAMc does not exist in the Arabidopsis genome, but is present in other Arabidopsis species and in Boechera. Hence, this gene was lost in A. thaliana after divergence from its congeners.

Analyses of nucleotide substitution patterns revealed that MAMI had accumulated an excess of non-synonymous nucleotide substitutions (i.e., substitutions that alter codon meaning) after the $M A M a$ duplication, indicating positive (Darwinian) selection. The biochemical characteristics of MAM1, MAM2 and MAMa were investigated to infer which properties of $M A M 1$ were targeted by positive selection. MAMa from A. lyrata and from Boechera stricta (a close relative of $B$. divaricarpa), MAM2 from the Arabidopsis accession Ler, and MAM1 from the accession Sorbo were heterologously expressed in Escherichia coli. Enzyme assays were carried out to investigate substrate specificity, ATP-, metal ion- and pH dependence (Benderoth et al. 2006). Major differences were found only for the enzymes' substrate specificities (Table 1). A. petraea and B. stricta MAMa, and Ler $M A M 2$ all utilized 4-methylthio-2-oxobutanoic acid (2C) for condensation with acetyl-CoA, but none accepted $\omega$-methylthio-2-oxoalkanoic acids with more than two methylene groups as a substrate. By contrast, Sorbo MAM1 accepted 4-methylthio-2-oxobutanoic acid (2C), 5-methylthio-2-oxopentanoic acid (3C) and, with low activity, also 6-methylthio-2-oxohexanoic 


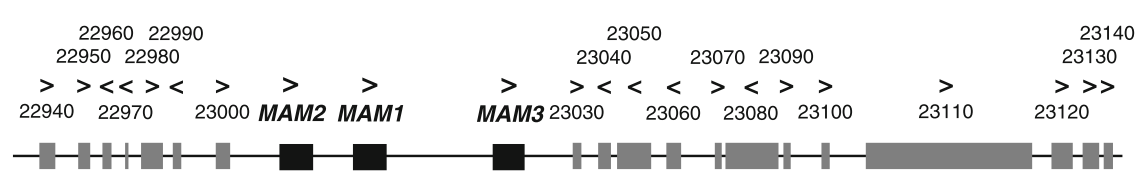

\section{A. thaliana}

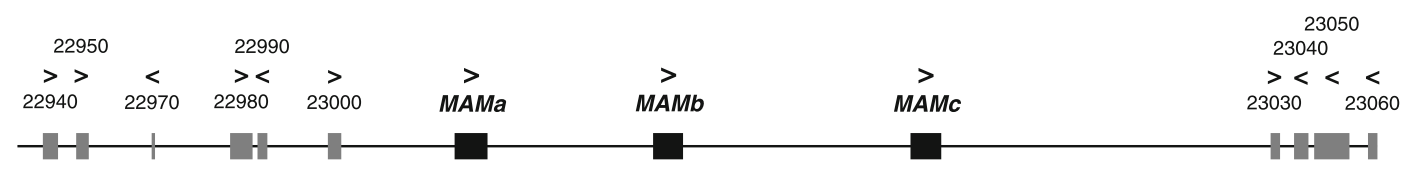

\section{A. lyrata}

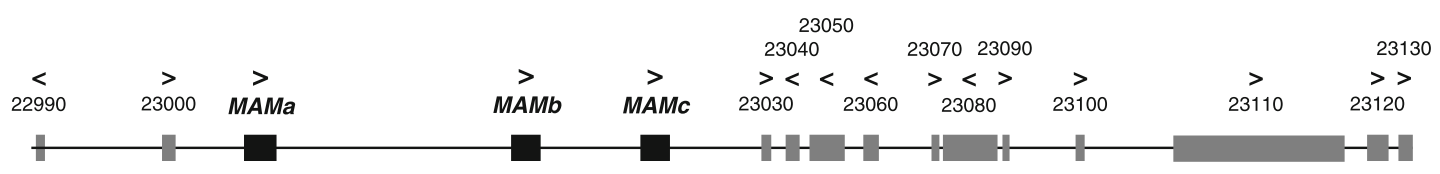

A. petraea

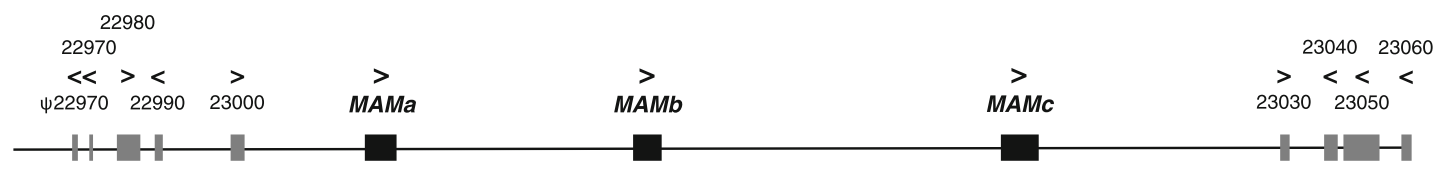

\section{A. cebennensis}

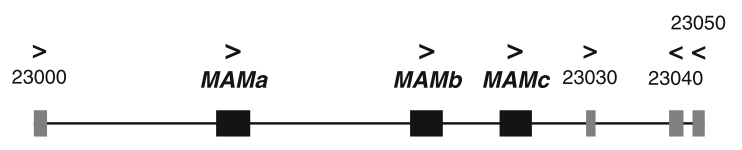

$10 \mathrm{kbp}$

\section{B. divaricarpa}

Fig. 9 Organization of the $M A M$ region in $A$. thaliana relatives. The $M A M$ cluster contains three genes (MAMa, $M A M b$ and $M A M c$ ) in A. lyrata, A. petraea, A. cebennensis and $B$. divaricarpa. The regions flanking $M A M$ are largely

acid (4C) as a substrate. Thus, MAMa and MAM2 function only in the first cycle of carbon chain extension, whereas MAM1 has acquired additional capacity to carry out condensation reactions in subsequent chain elongation cycles, equivalent to a biochemical neofunctionalization. MAM1 and MAM2 substrate specificities are matched by A. thaliana glucosinolate phenotypes. Accessions with a functional $M A M 1$ gene accumulate 4C glucosinolates, whereas accessions without a functional MAMI (but with a functional MAM2) generate mainly $3 \mathrm{C}$ aliphatic glucosinolates (Kroymann et al. 2003). Thus, gene duplication, biochemical neofunctionalization and positive selection account for the generation of diversity in the carbon chain lengths of aliphatic glucosinolates in A. thaliana.

The same processes are duplicated along the basal branches of the MAM gene tree (Fig. 3). MAMa, co-linear. For comparison, the archetypical configuration for A. thaliana is included. Numbers refer to AGI annotations (Arabidopsis Genome Initiative 2000), and the direction of transcription is indicated

$M A M b$ and $M A M c$ have originated by gene duplication events, and are functionally diversified. MAMa controls short-chain aliphatic glucosinolates (Benderoth et al. 2006) and $M A M b$ is, like its A. thaliana ortholog MAM3 (Textor et al. 2007; Knoke et al. 2008), presumably involved in the biosynthesis of long-chain aliphatic glucosinolates. However, the function of $M A M c$ is not yet known. Analyses of nucleotide substitution patterns indicate positive selection along the deep branches of the MAM tree, connecting MAMa, $M A M b$ and $M A M c$ (Benderoth et al. 2006). Hence, the same events that explain diversity in short-chain glucosinolates, i.e., gene duplication and biochemical neofunctionalization, driven by positive selection, appear to account for the diversification in aliphatic glucosinolate carbon chain lengths in general.

Dihomomethionine (4C) differs by only one methylene group from homomethionine (3C). At first 
glance, this seems to be a minor difference. But the consequences of this small chemical difference can be profound when taken within the context of the glucosinolate-myrosinase system. After methionine carbon chain elongation and glucosinolate core structure generation, enzymes encoded at other genetic loci act to modify the carbon chain (Kliebenstein et al. 2001a, b). These loci are, like MAM, also polymorphic in A. thaliana, i.e., they harbour alleles whose gene products have diverse biochemical activities and cause different modifications of the aliphatic carbon chain. Likewise, modifying proteins like ESP (Lambrix et al. 2001) and ESM1 (Zhang et al. 2006) can alter glucosinolate breakdown identity during myrosinase-catalyzed glucosinolate hydrolysis. Taken together, different combinations of alleles at glucosinolate biosynthesis and hydrolysis loci generate quite different blends of glucosinolate hydrolysis products (Kliebenstein et al. 2005), and these may, in turn, cause different responses in attacking insect herbivores and other enemies.

\section{Maintenance of glucosinolate diversity}

While gene duplication, neofunctionalization and positive selection contribute to the generation of metabolic diversity, these processes do not satisfyingly explain how and why this diversity is maintained. Positive selection on MAMI indicates that this gene has provided a fitness advantage to its carriers after duplication of an ancestral MAMa gene. MAM1 overrides MAM2 function, and MAM1 has retained the capacity to function in the first methionine carbon chain elongation cycle (Kroymann et al. 2003; Benderoth et al. 2006). Thus, the MAM2 is not required to sustain MAM1 activity. Why then is $M A M 2$ still present in A. thaliana?

It could be possible that MAM2 is in the process of becoming a pseudo-gene, but that the period after MAM1 neofunctionalization was too short for a complete degeneration of MAM2. For two reasons, this is not likely. First, estimates based on the analysis of nucleotide substitution rates in the Brassicaceae (Yang et al. 1999; Koch et al. 2001) suggest that the MAMa duplication occurred more than $10^{5}$ generations ago. Second, a degenerating gene is expected to accumulate nucleotide substitutions since selection no longer acts to eliminate deleterious mutations.
Mutations in open reading frames are called synonymous when the amino acid sequence remains unchanged and non-synonymous when the codon usage is altered. Because of the nature of the genetic code, a coding sequence has more non-synonymous than synonymous positions. Therefore, in a degenerating gene non-synonymous changes are more likely to occur than synonymous substitutions. But after correction for the number of non-synonymous and synonymous positions, the ratio between non-synonymous and synonymous changes in a degenerating gene is expected to be close to 1 . However, when MAM2 was compared to A. thaliana MAM1 or A. lyrata MAMa, MAM2 exhibited an excess of synonymous relative to non-synonymous changes, indicating purifying selection (Benderoth et al. 2006). In conclusion, MAM2 function appears to be preserved in A. thaliana.

What else could explain why MAM2 was retained in A. thaliana? Insect herbivory assays had shown that NILs with the Ler MAM2 performed ca. $17 \%$ better against $S$. exigua larvae than lines with the Col0 MAM1 (Kroymann et al. 2003). Thus, MAM2 can provide a selective advantage over MAM1 under certain conditions. In addition, secondary gene deletion events and exchange of sequence information between paralogous MAM1 and MAM2 loci have occurred frequently in the history of $A$. thaliana (Fig. 6). Deletion of $M A M 1$ or conversion of MAM1 into a $M A M 2$-like sequence both result in a switch from a $4 \mathrm{C}$ glucosinolate profile to a profile dominated by $3 \mathrm{C}$ glucosinolates. If such a switch occurred in a local population of plants that were otherwise genetically uniform, this could confer a-temporalselective advantage to the novel genotypes, provided that the local herbivore community was accustomed to a particular blend of glucosinolates. Since $A$. thaliana propagates mostly by selfing and local populations were founded frequently in the history of this species, such a scenario is not unlikely, and might account for the large proportion of derived genotypes at the MAM locus.

Furthermore, the composition of local herbivore communities varies temporally, with different classes of insects-specialists, generalists, and non-feeders (i.e., herbivorous insects not utilizing glucosinolatecontaining plants as a host)_occurring in different frequencies over time. This may lead to fluctuating selection on glucosinolate profiles, with periods during which phenotypes with a particular glucosinolate 
composition or with high glucosinolate levels increase in frequency, alternating with periods during which other types of glucosinolate profiles or low levels are advantageous. As a consequence, one would expect some form of equilibrium between different glucosinolate phenotypes. Indeed, among 51 Arabidopsis accessions whose glucosinolate profiles have been analyzed (Kliebenstein et al. 2001a; Kroymann et al. 2003; Pfalz et al. 2007), 29 produced aliphatic glucosinolates predominantly from homomethionine (3C) and 22 from dihomomethionine (4C). These data are not significantly different from the hypothesis that both phenotypes have equal frequencies in $A$. thaliana $\left(N=51 ; d f=1, \chi^{2}=1.27\right.$, n.s.). Also, two independent statistical tests of molecular population genetics found evidence for balancing selection acting on the MAM2 gene (Kroymann et al. 2003). Balancing selection refers to evolutionary scenarios that maintain more genetic variation in a population than expected under neutrality (Nordborg and Innan 2002). First, a positive Tajima's $D$ indicated significantly more intermediate frequency nucleotide polymorphisms segregating at MAM2 than expected (Tajima 1989). Second, a McDonald and Kreitman test (McDonald and Kreitman 1991) showed that too many amino acids segregated in A. thaliana MAM2, when compared to $M A M a$ from A. lyrata. However, this MAM2 polymorphism has no impact on glucosinolate identity (Kroymann et al. 2003; Benderoth et al. 2006), suggesting that non-neutrality at MAM2 is caused by selection on glucosinolate quantity and not quality, a hypothesis that remains to be tested.

While gene duplication, biochemical neofunctionalization and positive selection account for the generation of metabolic diversity at the MAM locus, secondary gene deletions, gene conversion and balancing selection appear to maintain biochemical diversity. Of course, deletion of MAM1 and conversion of MAM1 into a MAM2-like sequence are both one-way streets from an archetypical MAM2-MAM1$M A M 3$ configuration. Likewise, deletion of MAM2 or conversion of MAM2 into a MAM1-like gene both prevents future switches of chain-length phenotypes. Therefore, the Arabidopsis MAM locus appears to be in a process during which paralogous genes are being sorted among lineages, ultimately leading to plants that harbor, in combination with MAM3, either a $M A M 1$ or a MAM2 gene.

\section{Similarities and differences in chain-length variation between Arabidopsis and Brassica}

In Brassica oleracea and other members of the genus, aliphatic glucosinolates can be generated from homo-, dihomo- and trihomomethionine (Velasco and Becker 2000). As in A. thaliana, there is natural variation for homo- versus dihomomethionine-derived glucosinolates among different accessions. However, in contrast to A. thaliana, accumulation of homomethionine- and accumulation of dihomomethioninederived glucosinolates do not mutually exclude each other. Hence, B. oleracea accessions can produce aliphatic glucosinolates from homomethionine (3C), dihomomethionine (4C) or from homomethionine and dihomomethionine $(3 \mathrm{C}+4 \mathrm{C})$. This biochemical polymorphism is caused by variation at two different genetic loci, BoGSL-ELONG and BoGSL-PRO (Magrath et al. 1994; Li et al. 2001). BoGSL-ELONG harbors a typical MAM gene with ten exons (Fig. 4), and, in addition, a MAM pseudogene (Li et al. 2001; Gao et al. 2005). The B. oleracea MAM gene is closely related to MAM genes from other Brassicaceae (Fig. 3). Genetic data indicate that BoGSL$E L O N G$ is responsible for the generation of dihomomethionine-derived glucosinolates ( $\mathrm{Li}$ and Quiros 2002). However, B. oleracea MAM does not belong to any of the MAMa, $b$ or $c$ subclades found in $A$. thaliana and close relatives (Fig. 3). The second Brassica locus, BoGSL-PRO, contains a gene with a typical 12-exon IPMS structure (Fig. 4), and phylogenetic analyses support a close phylogenetic relationship with other IPMS genes (Fig. 3). This gene is supposedly involved in the generation of homomethionine-derived glucosinolates (Gao et al. 2006). This suggests that (i) the gene duplication events leading to MAMa, $b$ and $c$ occurred after Arabidopsis, Boechera and Brassica diverged from a common ancestor, (ii) the ability to utilize $3 \mathrm{C}$ precursors for carbon chain elongation evolved independently in Brassica and Arabidopsis and (iii) the Brassica MAM function responsible for generating 3C glucosinolates evolved de novo from a MAM progenitor gene. To confirm these hypotheses it will be necessary to sample further MAM (and also IPMS) genes from close and distant Arabidopsis and Brassica relatives and to analyse the biochemical properties of the encoded proteins. 


\section{Conclusions}

The composition of genes at the MAM locus varies between and within cruciferous species, causing substantial diversity in glucosinolate profiles. Different types of selection act on $M A M$ gene family members, and different factors account for generating glucosinolate variability and for maintaining this diversity. Comparative analyses suggest that particular MAM substrate specificities have evolved repeatedly in different genera of glucosinolate-producing plants. The model plant $A$. thaliana has been invaluable for making progress in dissecting the genetic, functional and ecological basis of glucosinolate diversity. These discoveries have already facilitated the identification and cloning of genes from the glucosinolate-myrosinase system in cruciferous species for which an ab initio approach proves much more difficult, such as crop plants or wild Arabidopsis relatives. Further functional and evolutionary studies, involving additional species from the Brassicaceae but also from the Capparaceae, the second large family in the Capparales order that is capable of using methionine homologs as glucosinolate precursors, will help to better understand the complexity and evolutionary dynamics of variation in plant secondary metabolism.

Acknowledgements We thank John D'Auria for critical reading of the manuscript and gratefully acknowledge financial support from the Max Planck Society and the DFG (grants KR2237/2-1, KR2237/2-2 and KR2237/2-3 to JK).

Open Access This article is distributed under the terms of the Creative Commons Attribution Noncommercial License which permits any noncommercial use, distribution, and reproduction in any medium, provided the original author(s) and source are credited.

\section{References}

Arabidopsis Genome Initiative (2000) Analysis of the genome sequence of the flowering plant Arabidopsis thaliana. Nature 408:796-815

Benderoth M, Textor S, Windsor AJ, Mitchell-Olds T, Gershenzon J, Kroymann J (2006) Positive selection driving diversification in plant secondary metabolism. Proc Natl Acad Sci USA 103:9118-9123

Campos de Quiros H, Magrath HCR, McCallum D, Kroymann J, Schnabelrauch D, Mitchell-Olds T, Mithen R (2000) Quantitative trait loci and the biochemical genetics of glucosinolate biosynthesis in Arabidopsis thaliana. Theor Appl Genet 101:429-437
Daxenbichler ME, Spencer GF, Carlson DG, Rose GB, Brinker AM, Powell RG (1991) Glucosinolate composition of seeds from 297 species of wild plants. Phytochemistry 30:2623-2638

de Kraker JW, Luck K, Textor S, Tokuhisa JG, Gershenzon J (2007) Two Arabidopsis thaliana genes (IPMS1 and IPMS2) encode isopropylmalate synthase, the branchpoint step in the biosynthesis of leucine. Plant Physiol 143: 970-986

Fahey JW, Zalcmann AT, Talalay P (2001) The chemical diversity and distribution of glucosinolates and isothiocyanates among plants. Phytochemistry 56:5-51

Falk KL, Vogel C, Textor S, Bartram S, Hick A, Pickett JA, Gershenzon J (2004) Glucosinolate biosynthesis: demonstration and characterization of the condensing enzyme of the chain elongation cycle in Eruca sativa. Phytochemistry 65:1073-1084

Field B, Cardon G, Traka M, Botterman J, Vancanneyt G, Mithen R (2004) Glucosinolate and amino acid biosynthesis in Arabidopsis. Plant Physiol 135:828-839

Gao M, Li G, McCombie RW, Quiros CF (2005) Comparative analysis of a transposon-rich Brassica oleracea BAC clone with its corresponding sequence in A. thaliana. Theor Appl Genet 111:949-955

Gao M, Li G, Potter D, McCombie RW, Quiros CF (2006) Comparative analysis of methylthioalkylmalate synthase (MAM) gene family and flanking DNA sequences in Brassica oleracea and Arabidopsis thaliana. Plant Cell Rep 25:592-598

Hagelstein P, Schultz G (1993) Leucine synthesis in spinach chloroplasts: partial characterization of 2-isopropylmalate synthase. Biol Chem Hoppe-Seyler 374:1105-1108

Haughn GW, Davin L, Giblin M, Underhill EW (1991) Biochemical genetics of plant secondary metabolites in Arabidopsis thaliana: the glucosinolates. Plant Physiol 97:217-226

Heidel A, Clauss M, Kroymann J, Savolainen O, Mitchell-Olds $\mathrm{T}$ (2006) Natural variation in MAM within and between populations of Arabidopsis lyrata determines glucosinolate phenotype. Genetics 173:1629-1636

Huelsenbeck JP, Ronquist F (2001) MRBAYES: Bayesian inference of phylogeny. Bioinformatics 17:754-755

Keurentjes JJB, Fu J, de Vos CHR, Lommen A, Hall RD, Bino RJ, van der Plas LHW, Jansen RC, Vreugdenhil D, Koornneef M (2006) The genetics of plant metabolism. Nat Genet 38:842-849

Kliebenstein DJ (2008) A quantitative genetics and ecological model system: understanding the aliphatic glucosinolate biosynthesis network via QTLs. Phytochem Rev. doi: 10.1007/s11101-008-9102-8

Kliebenstein DJ, Kroymann J, Brown P, Figuth A, Pedersen D, Gershenzon J, Mitchell-Olds T (2001a) Genetic control of natural variation in Arabidopsis glucosinolate accumulation. Plant Physiol 126:811-825

Kliebenstein DJ, Lambrix VM, Reichelt M, Gershenzon J, Mitchell-Olds T (2001b) Gene duplication in the diversification of secondary metabolism: tandem 2-oxoglutaratedependent dioxygenases control glucosinolate biosynthesis in Arabidopsis. Plant Cell 13:681-693

Kliebenstein DJ, Gershenzon J, Mitchell-Olds T (2001c) Comparative quantitative trait loci mapping of aliphatic, 
indolic and benzylic glucosinolate production in Arabidopsis thaliana leaves and seeds. Genetics 159: 359-370

Kliebenstein DJ, Kroymann J, Mitchell-Olds T (2005) The glucosinolate-myrosinase system in an ecological and evolutionary context. Curr Opin Plant Biol 8:264-271

Kliebenstein DJ, West MAL, van Leeuwen H, Loudet O, Doerge RW, St. Clair DA (2006) Identification of QTLs controlling gene expression networks defined a priori. BMC Bioinformatics 7:308

Knoke B, Textor S, Gershenzon J, Schuster S (2008) Modelling aliphatic glucosinolate diversity in Arabidopsis thaliana. Phytochem Rev (this issue)

Koch MA, Haubold B, Mitchell-Olds T (2001) Molecular systematics of the Brassicaceae: evidence from coding plastidic matK and nuclear Chs sequences. Am J Bot 88:534-544

Kroymann J, Donnerhacke S, Schnabelrauch D, Mitchell-Olds T (2003) Evolutionary dynamics of an Arabidopsis insect resistance quantitative trait locus. Proc Natl Acad Sci USA 100:14587-14592

Kroymann J, Mitchell-Olds T (2005) Epistasis and balanced polymorphism influencing complex trait variation. Nature 435:95-98

Kroymann J, Textor S, Tokuhisa JG, Falk KL, Bartram S, Gershenzon J, Mitchell-Olds T (2001) A gene controlling variation in Arabidopsis thaliana glucosinolate composition is part of the methionine chain elongation pathway. Plant Physiol 127:1077-1088

Lambrix V, Reichelt M, Mitchell-Olds T, Kliebenstein DJ, Gershenzon J (2001) The Arabidopsis epithiospecifier protein promotes the hydrolysis of glucosinolates to nitriles and influences Trichoplusia ni herbivory. Plant Cell 13:2793-2807

Li G, Quiros CF (2002) Genetic analysis, expression and molecular characterization of BoGSL-ELONG, a major gene involved in the aliphatic glucosinolate pathway of Brassica species. Genetics 162:1937-1943

Li G, Riaz S, Goyal S, Abel S, Quiros CF (2001) Inheritance of three major genes involved in the synthesis of aliphatic glucosinolates in Brassica oleracea. J Amer Soc Hort Sci 126:427-431

Lister C, Dean C (1993) Recombinant inbred lines for mapping RFLP and phenotypic markers. Plant J 4:745-750

Magrath R, Bano F, Morgner M, Parkin I, Sharpe A, Lister C, Dean C, Lydiate D, Mithen RF (1994) Genetics of aliphatic glucosinolates. I. Side chain elongation in Brassica napus and Arabidopsis thaliana. Heredity 72:290-299

Martin W, Herrmann RG (1998) Gene transfer from organelles to the nucleus: how much, what happens, and why? Plant Physiol 118:9-17

McDonald JH, Kreitman M (1991) Adaptive evolution at the Adh locus in Drosophila. Nature 251:652-654

Mithen R, Clarke J, Lister C, Dean C (1995) Genetics of aliphatic glucosinolates. III. Side chain structure of aliphatic glucosinolates in Arabidopsis thaliana. Heredity 74:210-215

Nordborg M, Innan H (2002) Molecular population genetics. Curr Opin Plant Biol 5:69-73

Pfalz M, Vogel H, Mitchell-Olds T, Kroymann J (2007) Mapping of QTL for resistance against the crucifer specialist insect herbivore Pieris brassicae in a new Arabidopsis inbred line population, $\mathrm{Da}(1)-12 \times \mathrm{Ei}-2$. PLoS ONE 2:e578

Purrington CB (2000) Costs of resistance. Curr Opin Plant Biol 3:305-308

Reichelt M, Brown PD, Schneider B, Oldham NJ, Stauber E, Tokuhisa J, Kliebenstein DJ, Mitchell-Olds T, Gershenzon J (2002) Benzoic acid glucosinolate esters and other glucosinolates from Arabidopsis thaliana. Phytochemistry 59:663-671

Rodman JE (1991a) A taxonomic analysis of glucosinolateproducing plants, part 1: phenetics. Syst Bot 16:598-618

Rodman JE (1991b) A taxonomic analysis of glucosinolateproducing plants, part 2: cladistics. Syst Bot 16:619-629

Rodman JE, Karol KG, Price RA, Sytsma KJ (1996) Molecules, morphology, and Dahlgren's expanded order Capparales. Syst Bot 21:289-307

Tajima F (1989) Statistical method for testing the neutral mutation hypothesis by DNA polymorphism. Genetics 123:585-595

Textor S, Bartram S, Kroymann J, Falk KL, Hick A, Pickett JA, Gershenzon J (2004) Biosynthesis of methionine-derived glucosinolates in Arabidopsis thaliana: recombinant expression and characterization of methylthioalkylmalate synthase, the condensing enzyme of the chain elongation cycle. Planta 218:1026-1035

Textor S, de Kraker JW, Hause B, Gershenzon J, Tokuhisa G (2007) MAM3 catalyzes the formation of all aliphatic glucosinolate chain lengths in Arabidopsis. Plant Physiol 144:60-71

Tian D, Traw MB, Chen J, Kreitman M, Bergelson (2003) Pleiotropic fitness cost of Rpml resistance in Arabidopsis thaliana. Nature 423:74-77

Velasco L, Becker HC (2000) Variability for seed glucosinolates in a germplasm collection of the genus Brassica. Genet Res Crop Evol 47:231-238

Wang S, Basten CJ, Zeng ZB (2001-2004) Windows QTL cartographer 2.5. Department of Statistics, North Carolina State University, Raleigh, NC. www.statgen.ncsu. edu.qtlcart/WQTLCart.htm

Wentzell AM, Rowe HC, Hansen BG, Ticconi C, Halkier BA, Kliebenstein DJ (2007) Linking metabolic QTLs with network and cis-eQTLs controlling biosynthetic pathways. PLoS Genet 3:e162

Whelan S, Goldman N (2001) A general empirical model of protein evolution derived from multiple protein families using a maximum-likelihood approach. Mol Biol Evol 18:691-699

Windsor AJ, Reichelt M, Figuth A, Svatoš A, Kroymann J, Kliebenstein DJ, Gershenzon J, Mitchell-Olds T (2005) Geographic and evolutionary diversification of glucosinolates among near relatives of Arabidopsis thaliana (Brassicaceae). Phytochemistry 66:1321-1333

Yang YW, Lai KN, Tai PY, Li WH (1999) Rates of nucleotide substitution in angiosperm mitochondrial DNA sequences and dates of divergence between Brassica and other angiosperm lineages. J Mol Evol 48:597-604

Zhang Z, Ober JA, Kliebenstein DJ (2006) The gene controlling the quantitative trait locus epithiospecifier modifier1 alters glucosinolate hydrolysis and insect resistance in Arabidopsis. Plant Cell 18:1524-1536 